\title{
Smoking: can we really make a difference?
}

\section{G Sutherland}

Heart 2003;89(Suppl II):ii25-ii27

The enormous health benefits of stopping smoking are now well established. Doctors have a vital role in motivating smokers and initiating quit attempts. The mainstay of National Health Service smoking cessation strategy should be the routine provision of brief opportunistic intervention in primary care, backed up by referral to a specialist smoking cessation service. There is an urgent need to increase substantially the numbers of smokers referred by general practitioners, other members of the primary care team, and those working in acute hospital trusts, to specialist smoking cessation services and for better channels of communication between the various agencies. Use of pharmacotherapy (nicotine replacement therapy or bupropion) in combination with behavioural support achieves higher cessation rates than either component alone and is the most effective way of helping smokers to stop. Smokers who quit often relapse and hence will need repeated help.

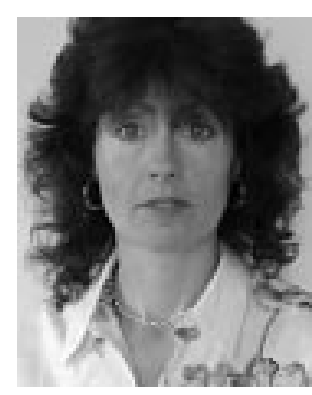

S mokers have roughly double the risk of ischaemic heart disease compared with nonsmokers and they have a two- to fourfold increased risk of sudden death. The effect of smoking on risk of myocardial infarction is greater in younger smokers. ${ }^{1}$ The cardiovascular benefits of stopping smoking are, however, now well established. The risk of coronary heart disease reduces rapidly after smoking cessation. The excess risk is reduced by about $50 \%$ after one year's abstinence and continues to decline gradually so that after $5-10$ years the risk is similar to that of a person who has never smoked. Stopping smoking reduces the risk of stroke to non-smoker level after five years.

The Royal College of Physicians has stated that nicotine addiction must be recognised as a major medical problem and that it is as strong as addiction to drugs such as cocaine and heroin -in some respects it actually exceeds those addictions. ${ }^{2}$ The power of nicotine addiction is demonstrated by the fact that about $50-70 \%$ of smokers relapse after a myocardial infarction, with nearly $40 \%$ of those who relapse doing so while they are still in hospital. ${ }^{3}$ Among all smokers, about $2 \%$ of unaided attempts to stop smoking result in abstinence at one year. ${ }^{2}$

Both the government and the National Health Service (NHS) are now taking smoking cessation seriously. The 1998 White Paper Smoking kills detailed the government's package of antismoking measures, which included the establishment of specialist smoking cessation services in the NHS. With the initial three year funding, challenging targets were set by the Department of
SE5 8AF, UK

g.sutherland@iop.kcl.ac.uk
Health for the number of smokers successfully quitting using the services. It is expected that funding will continue to be provided to keep the momentum going with the allocation to primary care trusts from 2003 onwards reflecting the high priority of smoking cessation within the NHS.

Updated UK guidelines on smoking cessation were published in 2000. ${ }^{5}$ These evidence based guidelines are endorsed by 25 professional bodies including the Royal College of Physicians, the Royal College of General Practitioners, and the Royal College of Nursing. Obstacles to full implementation of the guidelines include the perception (which is slowly changing) that smoking is not a medical problem; in addition, medical students are not taught the priority or practice of smoking cessation, existing clinicians have little or no training in smoking cessation, and some doctors, managers, and policy makers still give low priority to smoking cessation.

\section{PRIMARY CARE SMOKING CESSATION SERVICES}

The UK smoking cessation guidelines ${ }^{4}$ and the Royal College of Physicians' report ${ }^{2}$ advocate that the mainstay of NHS smoking cessation strategy should be the routine provision of brief opportunistic intervention in primary care. Primary care health professionals should be proactive in undertaking smoking cessation activities and they are ideally placed to do so as $80 \%$ of smokers visit their general practitioner (GP) at least once a year. Primary care intervention should be backed up by referral wherever possible to a specialist smoking cessation clinic. It is not realistic to expect primary care staff to have the time to provide the more intensive support needed by some smokers.

The guidelines emphasise the need to keep records up to date (table 1). At present, patients might only be asked about their smoking habits on first registration; annual updating of records is recommended. The guidelines recommend that all smokers should be advised to stop during routine consultations, preferably at least once per year, and help should be offered. The action taken then depends on the individual smoker and his or her motivation to stop smoking. Those who are "ready to quit" should be prescribed nicotine replacement therapy (NRT) or bupropion and then be referred to a specialist clinic if they are willing to attend. Attendance at a specialist clinic will achieve higher success rate and greater cost efficacy. Some smokers will not be "ready to quit" but it is not the job of the GP to try to force them to stop smoking-GPs do not have the time to motivate every smoker who is not interested. But

Abbreviations: GP, general practitioner; NHS, National Health Service; NICE, National Institute for Clinical Excellence; NRT, nicotine replacement therapy 
Table 1 Smoking cessation guidelines for primary care. Adapted from West et al, ${ }^{5}$ with permission

Ensure records of smoking status are kept up to date (annually) General practitioners and other health care professionals to advise smokers to stop during routine consultations and offer help "Ready to quit"

- Prescribe NRT or bupropion "Not ready to quit"

- Provide information-repeat at later stage

Primary care staff should receive training to deliver opportunistic advice and to give accurate advice on using medications

NRT, nicotine replacement therapy

they are able to provide brief information about the individual's risk of smoking and benefits of quitting, maybe in the form of a leaflet, and can then repeat the advice to stop smoking the next time the person visits.

The use of pharmacological treatments (NRT or bupropion) has been shown in numerous clinical trials to improve the rate of smoking cessation. Use of pharmacotherapy in combination with behavioural support achieves a higher cessation rate than either component alone, and is the most effective way of helping smokers to stop.

NRT and bupropion are effective, even in patients with cardiovascular disease. The National Institute for Clinical Excellence (NICE) assessed these treatments in March $2002^{6}$ and supported their use as first line treatments for virtually all smokers who wish to stop, with no minimum cigarette consumption before treatment is recommended. A NICE meta-analysis of NRT from 97 randomised controlled trials involving 38000 smokers who were followed for six months after starting treatment showed an odds ratio of smoking cessation of NRT versus placebo of 1.74 (95\% confidence interval 1.64 to 1.86 ). For bupropion, a meta-analysis of 10 randomised controlled trials involving 3800 smokers, followed for six or 12 months, showed an odds ratio for smoking cessation versus placebo of 2.16 (95\% confidence interval 1.51 to 3.10$).^{6}$

There is a clear dose-response relationship between success at quitting and amount of behavioural support received. ${ }^{7}$ Since NRT approximately doubles success rate regardless of the amount of adjunctive support, increasing the amount of support increases the efficacy of NRT. Future studies are likely to show a similar result for bupropion. Patient characteristics, such as dependence level, motivation, mental health status, and age are also related to outcome. The success rate tends to be higher in older patients and those who are less nicotine dependent. The results with specific patient groups using these treatments are consistent with the overall efficacy-that is, roughly a doubling of the chance of quitting. However, lower success rates are often found in cardiovascular patients and in respiratory patients. The fact that these patients continue to smoke despite the obvious health effects caused by smoking indicates that they are highly addicted and thus likely to need more intensive help.

\section{POTENTIAL FOR SUCCESS WITH PRIMARY CARE INTERVENTION}

Studies have shown that many doctors perceive their efforts to assist smokers as ineffective and potentially damaging to the doctor-patient relationship. However, the data do not support such pessimism. For example, a typical three GP practice in the UK will have around 1400 adult smokers. It is estimated that of these, 951 will want to stop, 1100 will have tried to stop, 600 want to stop in the next year, and 500 try every year. Therefore, there is a great deal of interest in stopping smoking and many efforts to do so among smokers in primary care. The common view that smokers take little notice of doctors' advice to stop smoking is also unfounded. Many patients do attempt to quit

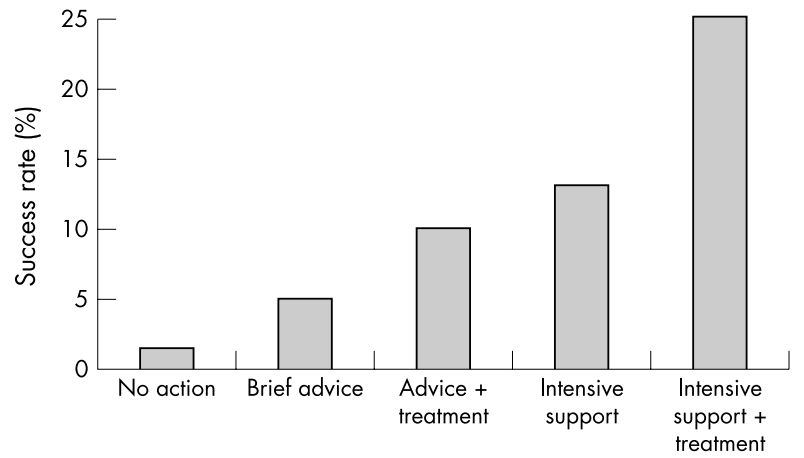

Figure 1 Expected one year success rates from different interventions (biochemically validated abstinence).

when a GP advises them to do so. ${ }^{89}$ Unfortunately doctors perceive that their advice has no effect as many smokers fail in their attempts because they are poorly planned, and do not involve either specialist behavioural support or appropriate pharmacotherapy. The important point is that the GP's advice motivated them to try to quit. The challenge is to harness the interest and motivation to quit, inspired by a doctor's advice, by ensuring smokers are aware of and use specialist support and medication, wherever possible.

Expected one year success rates (continuous abstinence) for smoking cessation are shown in fig $\mathrm{l}$. With no advice or support from a doctor or other health care professional only $1-2 \%$ of smokers will stop smoking and remain abstinent over the next year; with three minutes' advice from a doctor the success rate will be $5 \%$, and adding pharmacotherapy can increase this to $10 \%$. The success rate increases further when intensive behavioural support is provided from a specialist clinic, and intensive support plus pharmacotherapy can lead to $25 \%$ success rates at one year. ${ }^{7}$

A national GP attitudes survey in 2002 showed that fewer than $50 \%$ of GPs saw either NRT or bupropion as having high priority in their drug budget (unpublished data). Some GPs hold the view that the costs of trying to help people stop smoking, in terms of medications and professional time, make it an expensive intervention when so many patients fail in their cessation attempt. However, smoking cessation using NRT or bupropion is one of the most cost effective interventions in the NHS, ranging from about $£ 250$ (for brief advice from a GP) to $£ 1000$ (for a specialist smokers clinic) per discounted life year saved. This compares favourably with the costs for other interventions in cardiovascular prevention. Aspirin, given for secondary prevention, costs $£ 7750$ per life year saved. ${ }^{10}$ For statins, the cost efficacy figure varies with different analyses ${ }^{11-13}$ from $£ 5400$ to $£ 13000$, yet the NHS spends around 10 times as much on statins as it does on smoking cessation treatments. It should also be borne in mind that successful smoking cessation can hugely reduce the need for statin prescribing, by an estimated $80 \%$, as a result of the reduction of an individual's level of cardiovascular risk on quitting. ${ }^{14}$

\section{CONCLUSION}

To improve success in smoking cessation, primary health care professionals should systematically and repeatedly advise smokers to stop and more smokers should be persuaded to attend specialist cessation services, especially heavy smokers and those with smoking related disease. There is a need for better coordination of primary and secondary care efforts with the specialist clinics, and for these clinics, which have been built up successfully over the past three years or so, to be funded on a permanent basis in order to avoid losing experienced personnel. There should be wider use of pharmacotherapies as these significantly enhance long term success rates. Finally, it is also important to recognise that tobacco 
addiction is a chronic relapsing disorder, and many smokers who quit in the short term will relapse and hence will need repeated help, as is the case for other addictive disorders.

\section{ACKNOWLEDGEMENTS}

The author would like to acknowledge the assistance of Professor John Britton, Professor of Respiratory Medicine, City Hospital, Nottingham, UK.

\section{REFERENCES}

1 Parish S, Collins R, Peto R, et al. Cigarette smoking, tar yields and non-fatal myocardial infarction: 14000 cases and 32000 controls in the United Kingdom. BM J 1995;311:471-7.

2 Royal College of Physicians of London. Nicotine addiction in Britain: a report of the Tobacco Advisory Group of the Royal College of Physicians. London: Royal College of Physicians; 2000.

3 Bigelow GE, Rand CS, Gross J, et al. Smoking cessation and relapse among cardiac patients. In: Relapse and recovery in drug abuse. NIDA Research Monograph 72. Rockville, Maryland: US Department of Health \& Human Services 1986:167-71.

4 Secretary of State for Health. Smoking kills: a white paper on tobacco. London: Stationery Office, 1998.

5 West R, McNeill A, Raw M. Smoking cessation guidelines for health professionals: an update. Thorax 2000;55:987-99.
6 National Institute for Clinical Excellence. Guidance on the use of nicotine replacement therapy (NRT) and bupropion for smoking cessation. London: NICE, 2002.

7 Fiore MC, Bailey WC, Cohen SJ, et al. Treating tobacco use and dependence: clinical practice guideline. Rockville, Maryland: US Department of Health and Human Services, Public Health Service, 2000.

8 Russell MAH, Wilson C, Taylor C, et al. Effect of general practitioners' advice against smoking. BM 1979;2:231-5.

9 Kreuter MW, Chheda SG, Bull FC. How does physician advice influence patient behavior? Evidence for a priming effect. Arch Fam Med 2000;9:426-33

10 Gaspoz JM, Coxson PG, Goldman PA, et al. Cost effectiveness of aspirin, clopidogrel, or both for secondary prevention of coronary heart disease. N Engl J Med 2002;346:1800-6.

11 Caro J, Klittich W, McGuire A, et al. The West of Scotland coronary prevention study: economic benefit analysis of primary prevention with pravastatin. BMU 1997;315:1577-82.

12 Jonsson B, Johannesson M, Kjekshus J, et al. Cost-effectiveness of cholesterol lowering. Results from the Scandinavian simvastatin survival study. Eur Heart J 1996;17:1001-7.

13 Ebrahim S, Davey Smith G, McCabe C, et al. What role for statins? A review and economic model. Health Technology Assessment 1999;3(19).

14 Muir J, Fuller A, Lancaster T. Applying the Sheffield tables to data from general practice. Br J Gen Pract 1999;49:218-9. 